\title{
ÁREA DE IMPACTO DE LA COMUNICACIÓN POLÍ- TICA: ESTUDIO DE CASO (ELECCIONES ESPAÑOLAS 2004)
}

\author{
IMPACT AREA OF POLITICAL COMMUNICATION: CASE \\ STUDY (SPANISH ELECTIONS 2004)
}

\author{
Fermín Bouza Álvarez \\ Universidad Complutense \\ Raquel Rodríguez Díaz \\ Universidad Rey Juan Carlos \\ raquel.rodriguez@urjc.es
}

\section{Resumen}

Este trabajo recoge los principales resultados de una investigación centrada en el comportamiento electoral de las elecciones generales españolas de 2004, celebradas el 14 de marzo, pocos días después de los atentados terroristas de los trenes de Atocha del 11 de marzo. Partiendo de la hipótesis del área de impacto de la comunicación política, se analizan los temas más importantes para la opinión pública a lo largo de la legislatura 2000-2004 y con especial atención los meses previos a las elecciones. Unas elecciones marcadas por los atentados en los que los temas terrorismo, paro y vivienda ocupaban los tres problemas más importantes para los españoles tanto en la agenda pública como en la agenda personal, convirtiéndolos en temas clave de receptividad en la comunicación política.

Palabras clave: Agenda pública, agenda personal, agenda setting, elecciones y España.

\section{Summary}

This work gathers the main results of a study which focused on voter behaviour in the Spanish General Elections in 2004, held on the 14th March, only a few 
days after the terrorist bombings of trains in Atocha Station on the 11th of March. Using the hypothesis of the impact area of political communication, we have anaylsed the most important issues for public opinion during the 20002004 legislature, paying attention to the months leading up to the elections. The elections were marked by the bombings in which the issues of terrorism, unemployment and housing were the three most serious problems faced by the Spanish population according to both the public and the personal agenda, thus making them keys issues interested in political communication.

Keywords: Public agenda, personal agenda, agenda-setting, elections and Spain.

\section{INTRODUCCIÓN}

El presente artículo es el resultado de la comunicación presentada por Fermín Bouza y Raquel Rodríguez en el congreso internacional de la International Communication Association (ICA) celebrado en Nueva York en el año 2005, en un panel monográfico dedicado a las relaciones entre la comunicación y el terrorismo. Este trabajo que llevaba como título original "Examining the Spanish Public Agenda, Personal Agenda and "Impact Area" between March 11 to March 14, 2004" no llegó a publicarse como artículo, pese a que nos quedaba pendiente el hacerlo. He considerado importante sacar a la luz en esta revista uno de los conceptos más relevantes con los que contribuyó el profesor Bouza al ámbito académico de la comunicación política: el área de impacto. Este concepto se centra en la búsqueda de interpretaciones científicas de las opiniones conjuntas que expresamos los ciudadanos cuando diferenciamos entre la agenda pública y la agenda personal.

Retomamos en esta introducción algunos elementos teóricos extractados del artículo que dio nombre al concepto del área de impacto de la comunicación política (Bouza, 2004) que son esenciales para que el lector comprenda el estudio de caso en su conjunto, ya que en el texto original de la ICA no se incluyeron. En una versión en castellano del artículo de Bouza (2004) se señalaba en su resumen, publicado en la página personal del profesor $^{1}$, que:

\footnotetext{
${ }^{1}$ Página personal del Catedrático de Opinión Pública Fermín Bouza Álvarez, Departamento de Sociología VI, Facultad de Ciencias de la Información de la Universidad Complutense.

http://pendientedemigracion.ucm.es/info/socvi/BOUZA/NUEVA1/Textos/impactesp.pdf (visita realizada el 7 de julio de 20017).
} 
Este artículo es una reflexión sobre los cambios mediáticos y su repercusión en la Política y en la Comunicación Política, y quiere ser también una interpretación histórico-natural de las determinaciones de los medios de comunicación: el ser humano siempre ha estado sometido a oscuras fuerzas de extraordinaria influencia colectiva, y siempre ha salido adelante en su autonomía. En última instancia, es esa fuerza de la opinión la que ha ido preparando el camino de la Democracia y la que intenta preservarla libre en medio de las presiones más indeseables. Se propone también, a efectos de mejorar el conocimiento de las relaciones de los individuos con los intereses públicos, un análisis más detallado de lo que se llama el área de impacto de la Comunicación Política, que es el lugar teórico en que los individuos integran sus intereses personales (agenda personal) y sus interese públicos (agenda pública).

Desde un marco teórico en el que se recogen diversos aspectos de los sistemas democráticos, numerosos autores señalan que los medios de comunicación desempeñan funciones fundamentales, desde diferentes perspectivas y por diferentes motivos en los procesos de comunicación política (Swanson, 1995; Shulz, 1999; Blumler y McQuail, 1969; McCombs, 2004). Y otros textos resaltan, especialmente, la capacidad de la televisión e Internet como creadores de "un nuevo mundo de comunicación política global" (DeLuca y Peeples, 2002). Los individuos conocen de las actuaciones de los partidos y los políticos porque la prensa informa y opina sobre ellos, posicionando temas en la agenda mediática con enfoques (frames) (Entman, 1993; 2004) o marcos de referencia claros y precisos que ayudan a simplificar e interpretar el complicado mundo de la realidad.

En este sentido, los individuos aprenden de los medios a otorgar una importancia parecida a la cobertura que se ofrece de un tema informativo. Así lo evidencian los estudios de agenda-setting (McCombs y Shaw, 1972; McCombs, 2004), mostrando que, habitualmente, cuando un tema ocupa una mayor presencia en la agenda mediática, ese tema aumenta en la agenda pública, entendida ésta como reflejo de la opinión pública.

La implicación de los individuos en la agenda pública, que ellos mismos definen como colectivo es importante pero no absoluta. Bouza (2004) establece que los individuos mantienen diferentes conjuntos de intereses personales que se separan, en cierta medida, de los intereses públicos, generales o colectivos más presentes en la agenda pública. Esta diferenciación entre el conjunto de temas que los individuos mencionan como problemas para el país y los suyos personales (agenda personal) conlleva una diferenciación de intereses - objeto de análisis en este artículo-, haciendo posible una intersección de temas comunes entre la agenda pública y la personal, expresados como área de impacto (Figura A). 


\section{Figura A: Área de Impacto}

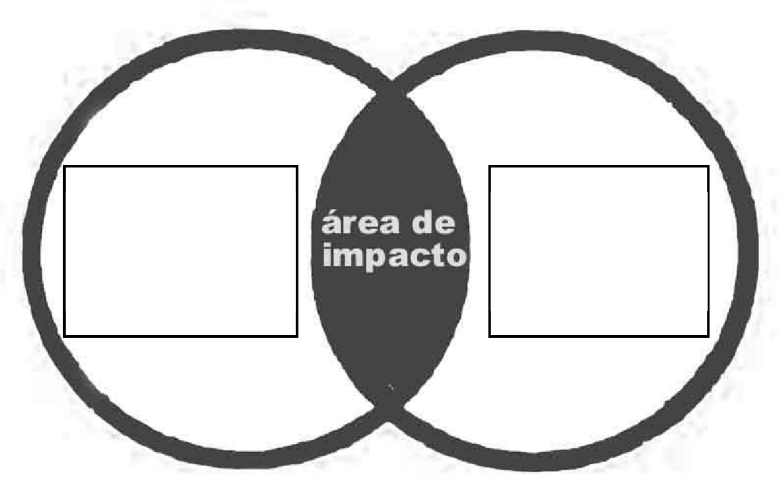

Fuente: Bouza, F. (2004): "The Impact Area of Political Communication: Citizenship Faced with Public Discourse", International Review of Sociology-Revue Internationale de Sociologie, Vol. 14, No. 2.

En esta dirección y, empleada de manera instrumental para detectar el grado de receptividad en la comunicación política que tienen los individuos, la definición del área de impacto se plantea como (Bouza, 2004: 250):

Aquella área temática más sensible a la comunicación pública en general y a la comunicación política en particular, porque es el área en la que el individuo siente una clara coincidencia entre el país y él mismo: una agenda mixta que tiene la fuerza de lo general y lo particular, y por ello mismo parece esa agenda por la que el individuo se siente más inclinado a presionar, al tiempo que es más receptivo a toda comunicación que se haga sobre ese bloque temático mixto. Podemos definir operativamente el área de impacto como la coincidencia mayor.

\section{PROCESO ELECTORAL: ELECCIONES ESPAÑOLAS 2004}

El trabajo que presentamos forma parte del desarrollo de un proyecto de investigación interdisciplinar financiado por el Ministerio de Ciencia y Tecnología español y centrado en comportamiento electoral ${ }^{2}$. Los resultados que ofrecemos son sólo una muy pequeña parte de una investigación aún en proceso que pretende complementarse con aspectos vinculados a la ciencia política, la agenda de los medios de comunicación y al propio análisis demoscópico de la evolución del voto de los españoles en las últimas legislaturas ${ }^{3}$. Aquí nos centraremos

\footnotetext{
2 Proyecto de Investigación financiado por el Ministerio de Ciencia y Tecnología: "Voto racional y control en la democracia representativa: Criterios de evaluación de la gestión del gobierno y comportamiento electoral. El caso español 1986-2004". Investigador responsable: Fermín Bouza Álvarez (BSO2000-0747-C02-01).

3 Algunos ejemplos de trabajos vinculados directa o indirectamente o herederos de este primer proyecto de investigación, al que se sumaron otros, son: González, J. J. (2002), Las elecciones generales del 2000. Voto ideológico / voto racio-
} 
en algunos de los aspectos a tener en cuenta para entender el contexto en el que se desarrollaron las elecciones generales españolas del 14 de marzo de 2004. Unas elecciones que contaron con la irrupción y conmoción de los atentados terroristas del 11 de marzo en la ciudad de Madrid, provocando un gran debate pre y post electoral, algunos ejemplos de autores así lo recogieron desde diferentes disciplinas (Álvarez de Toledo, 2004; Malalana, 2008; Lago y Montero, 2004; Michavila, 2005; Olmeda, 2005; Rodríguez (2016); Sampedro (2005); Santamaría (2004); Vara, Rodríguez, Giménez y Díaz, 2006).

El estudio comienza con el análisis de los temas que los españoles consideraban que eran los más importantes para el país a partir del mes de septiembre del 2000, coincidiendo con el año de inicio de la segunda legislatura del Partido Popular (PP) en el Gobierno con José María Aznar como presidente. Dichos temas los agruparemos en la agenda pública y veremos la evolución de tales temas que la opinión pública consideró los problemas más importantes durante la última legislatura del gobierno PP. A ello añadiremos la que nosotros llamamos agenda personal, esto es, los temas (problemas) que más preocupaban a los ciudadanos con relación a si mismos. Desde esta perspectiva, ubicamos el trabajo bajo el marco del estudio de la agenda de los ciudadanos, tanto pública como personal, acercándonos a algunos de los temas tradicionales del enfoque de la teoría de la Agenda Setting (McCombs y Shaw, 1972).

Al inicio de la legislatura (2000), el Partido Popular (PP) gobernaba con mayoría absoluta, y a medida que avanzaba el tiempo la distancia con su principal rival en la oposición, el Partido Socialista (PSOE), se fue reduciendo, llegando en el mes de enero-febrero de 2004 a una diferencia en intención directa de voto de 3,4 \% con respecto al PSOE, según el estudio preelectoral del Centro de Investigaciones Sociológicas (CIS)4. Tan sólo unos días antes de las elecciones y los atentados, los sondeos daban diferentes resultados para la intención de voto

nal, Revista Internacional de Sociología, 32, 7-33; Rodríguez. R. (2005). Miedo post 11-M y terrorismo en España. Recerca: revista de pensament $i$ anàlisi, (5), 127-142. Rodríguez, R. y Bouza, F. (2007). La inseguridad ciudadana en las agendas pública y personal de los españoles (2000-2004) / Citizen Insecurity in the Public and Private Agenda of the Spanish Population (2000-2004). Política y Sociedad, 44(3), 183-196. González, J. J. y Bouza F. (2009). Las razones del voto en la España democrática, 1977-2008 (Vol. 296). Madrid: Los libros de la Catarata; Rodríguez, R. y Castromil, A. R. (2010). La circulación social de los encuadres periodísticos en tiempo de campaña electoral: Transmisión, influencia y atribución de responsabilidad. Zer-Revista de Estudios de Comunicación, 15 (29), 193-212; Castromil, A. y Rodríguez, R. (2011) Terrorismo con y sin tregua. Políticos, ciudadanos y medios de comunicación. Revista TELOS (87), 46-56; Bouza, F., González, J. J., Rodríguez, R., Castromil, A. (2008). “Voto racional y agenda mediática. Propuesta de seguimiento de la legislatura a través de grupos experimentales". En M. J. Canel y M. Gurrionero). Estudios de comunicación política. Libro del año 2008 (pp. 501-520). Madrid: Servicio de publicaciones de la Universidad Complutense.

\footnotetext{
4 Estudio no 2.555, enero-febrero 2004. Centro de Investigaciones Sociológicas (CIS). http://www.cis.es/cis/export/sites/default/_Archivos/Marginales/2540_2559/2555/Es2555mar.pdf (visita realizada el 7 de mayo de 20017).
} 
directa o estimada. El Instituto Noxa (empresa más próxima al PSOE, pero trabajando para el diario La Vanguardia, con una tendencia conservadora) pronosticaba la victoria del PP por un margen de un 2,25 \% (41,4\%/39,2\%) de intención estimada de voto frente a otras empresas demoscópicas que daban una intención estimada de voto más amplia (entre 5 y 9,5 puntos de porcentaje). La intención directa de voto de Noxa era de 31,2\% para el PP, y 30,9\% para el PSOE. Es decir, la intención directa de voto daba una diferencia (CIS -enero/febrero-: 3,4; Noxamarzo-: 0,3) muy inferior a la estimada. Habría que añadir (más adelante están los datos) que la empresa TNS-Demoscopia (más próxima a medios conservadores, como el diario $A B C$, para el hacía sondeos electorales) daba una diferencia en intención directa de voto a favor del PSOE (el 10 de marzo, víspera de las bombas) de 1\% (23,9\% el PP, y 24,9\% el PSOE). Estos datos nos indican la magnitud del problema de intentar reconstruir la dinámica demoscópica de esos días. Pero sí podemos hablar de comunicación y gestión de la comunicación.

Durante la legislatura 2000-2004, diferentes temas fueron tomando relevancia y presencia como problemas para los ciudadanos, sirviendo para medir las diversas políticas que fue adoptando el ejecutivo para resolverlas. El aumento de la precariedad en el empleo, una huelga general, la inmigración y la inseguridad ciudadana, son algunos de los temas que más afectaron al PP durante la última etapa. A ello se sumó la implicación de España en la Guerra de Irak, con una fuerte oposición de la ciudadanía, un aumento vertiginoso de la subida de los precios de la vivienda y la catástrofe ecológica del petrolero Prestige. Como pilares de apoyo del gobierno del PP estaban un cierto saneamiento de las arcas del Estado, el descenso del desempleo y la lucha contra el terrorismo con el debilitamiento de ETA. Ante esta situación político-electoral, presentamos la evolución de los temas importantes desde la percepción del público, entendido sobre todo como votante, y enunciándolo bajo el epígrafe de agenda pública y personal.

\footnotetext{
${ }^{5}$ La Vanguardia, 7 de marzo de 2004. http://hemeroteca.lavanguardia.com/preview/2004/03/07/pagina1/33651159/pdf.html (visita realizada el 7 de mayo de 20017).
} 


\section{LOS TEMAS QUE MÁS PREOCUPABAN A LOS ESPAÑOLES 2000-2004.}

Los datos ofrecidos en las dos agendas analizadas proceden del seguimiento de los diversos temas a lo largo de la legislatura en los barómetros del CIS (septiembre 2000 - mayo 2004). En los estudios de Agenda-Setting se llama Agenda Pública a la respuesta que dan los sujetos de una encuesta a la pregunta del cuestionario que en España (CIS) se formula así: Para empezar, ¿cuáles son, a su juicio, los tres problemas principales que existen actualmente en España? (Respuesta espontánea). (Multirrespuesta: máximo tres respuestas). De igual modo, denominamos aquí Agenda Personal (o Público-Personal) (Bouza, 2004) a la respuesta que dan los sujetos de una encuesta a la pregunta del cuestionario que en España (CIS) se formula así: ¿Y cuáles son los tres problemas que, a Ud., personalmente, le afectan más? (Respuesta espontánea). (Multirrespuesta: máximo tres respuestas).

\section{Gráfico no $\mathbf{n}^{\mathbf{1}}$}

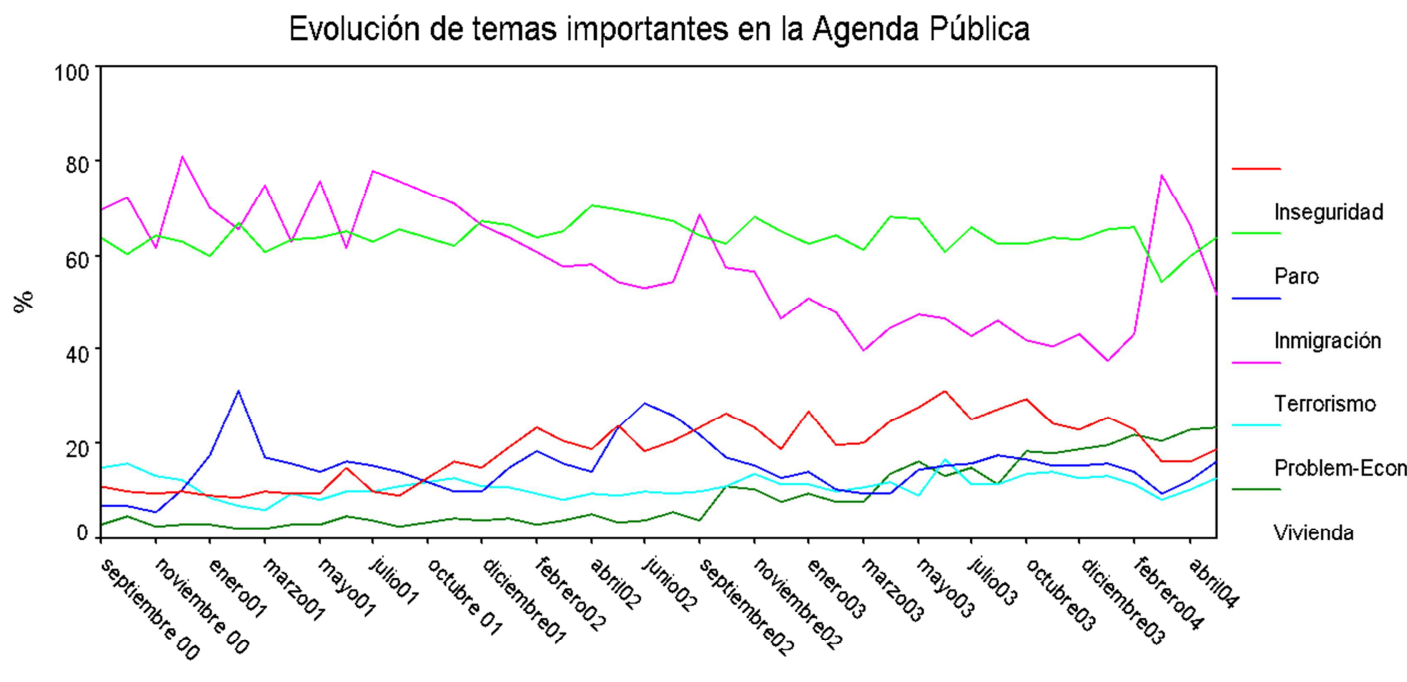

MES.AÑO (septiembre 2000 - mayo 2004)

Elaboración Propia. Fuente: CIS

En el Gráfico no1 se puede observar que el paro y el terrorismo han sido los temas "estrella" en la agenda pública, ocuparon siempre los porcentajes más destacados y no han dejado de estar en el primer o segundo lugar. En la medida en la que el paro ha ocupado el primer lugar (muchas más veces que el terrorismo), el terrorismo ha pasado al segundo y viceversa, según los acontecimientos del momento. Por lo tanto, el paro ha sido el tema de mayor preocupación, con diferencia, para los españoles.

La dominancia en la agenda pública del paro y terrorismo sólo ha dejado lugar para problemas o temas a ubicar desde la tercera posición. En este tercer lugar, 
encontramos mucha más fluctuación de temas y relevancia, en cuanto al porcentaje de los mismos. En el Gráfico nำ1 se aprecia que los problemas de mayor presencia y continuidad en la agenda, a excepción de paro y terrorismo, han sido inseguridad ciudadana, inmigración, vivienda y problemas económicos. En septiembre de 2000 tanto la inseguridad como la inmigración tienen una relativa visibilidad en la agenda, sus porcentajes son del 11\% y 6,7\% respectivamente duplicándose en febrero de 2004 y llegando así la inseguridad a un 22.9\% (ocupando el 3 lugar) y la inmigración un 13,9\%. Del mismo modo, la vivienda va sumando presencia y ganando posiciones a medida que se desarrolla la legislatura. En septiembre de 2000 comienza con un 3\% ocupando ese codicioso tercer lugar en marzo de 2004 con un $21,8 \%$, después de los atentados (Gráfico n-2). Igualmente, los problemas económicos (enunciados así, como "problemas económicos" porque también existen bajo otras formas como el paro, etc.) se mencionan constantemente en la agenda, además de tener una presencia considerable en porcentaje a lo largo de los cuatro años.

\section{Gráfico no 2}

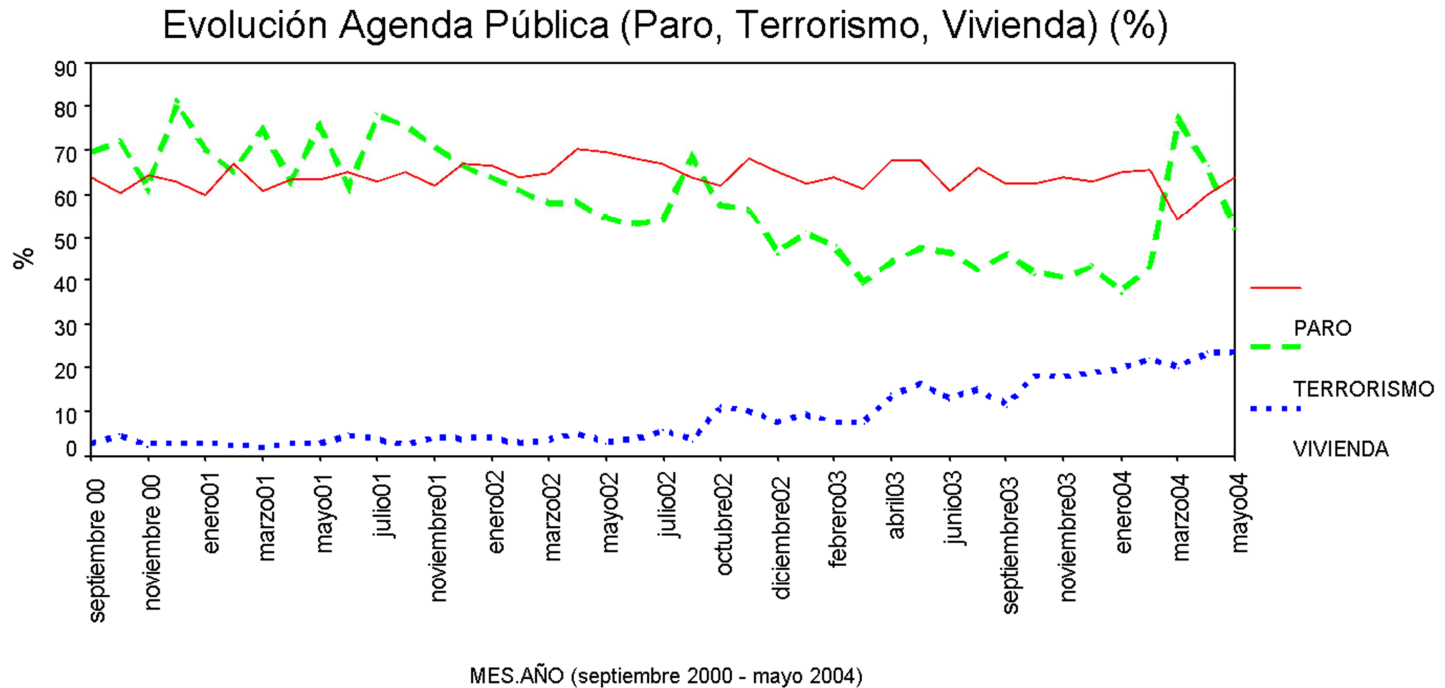

Elaboración Propia. Fuente: CIS

Mientras esto ocurre en la agenda pública, en la agenda personal pasan otras cosas y son otros los temas que más preocupan, aunque nosotros sólo nos centraremos en paro, terrorismo, vivienda y problemas económicos (véase el Gráfico n⿳1) ya que en los meses próximos a las elecciones estos fueron los que más despuntaron tanto en la agenda pública como en la personal (Gráfico nㅜㅜ). En este gráfico los porcentajes otorgados en la agenda personal son menores ya que están vinculados a una diferente distribución que en la agenda pública, aunque ello no afecta a los rangos, posiciones o lugares mostrados en el trabajo. 


\section{Gráfico nㅜㅇ}

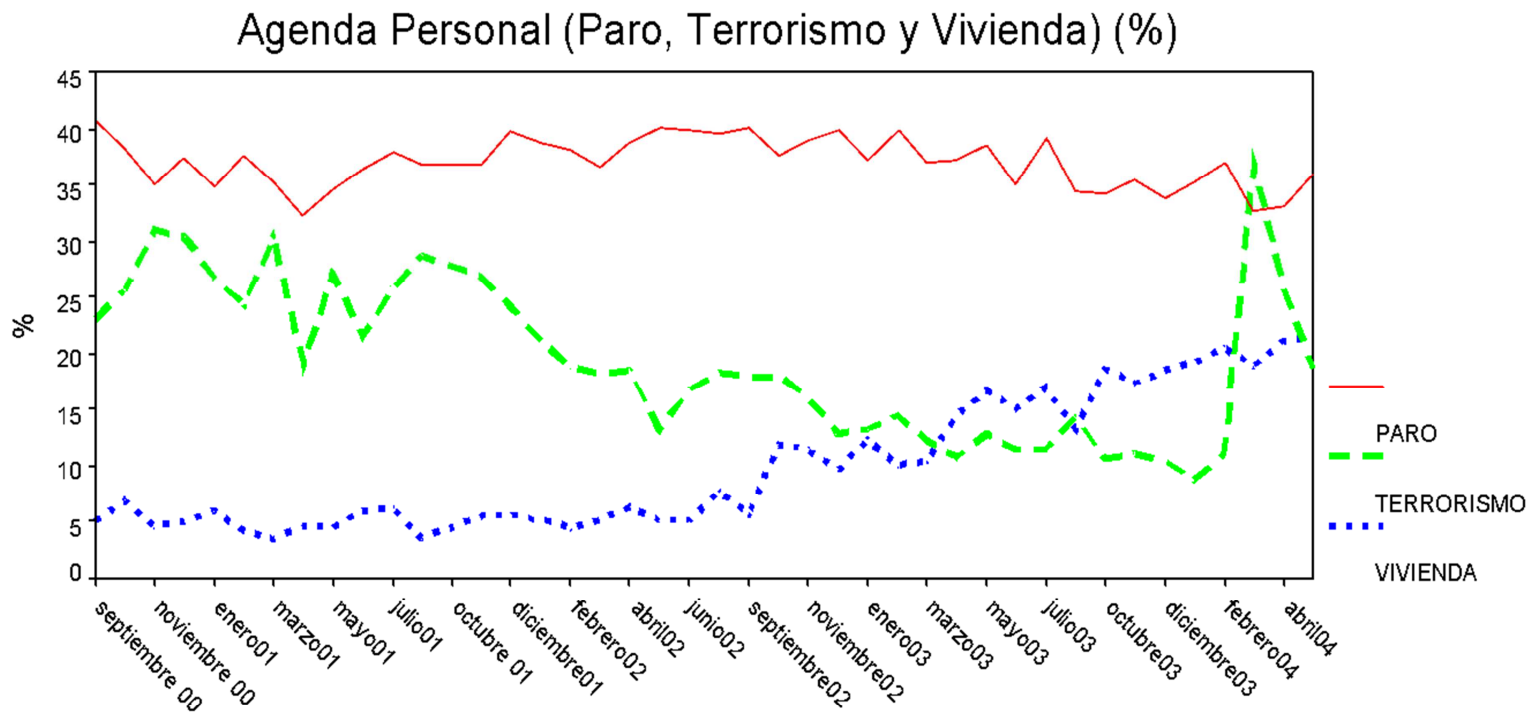

MES.AÑO Septiembre 2000 - Mayo 2004

Elaboración Propia. Fuente: CIS

Se puede observar en la agenda personal cómo el paro ha sido siempre el tema número uno, y nunca ha sido desbancado en el periodo analizado a excepción del mes de marzo de 2004, ocupando su lugar el terrorismo. Sin embargo, este último tema (terrorismo) ha pasado del segundo lugar en septiembre de $2000(23 \%)$ al sexto puesto en enero de 2004 (8,8\%). Por el contrario, mientras esto sucedía, la inseguridad ciudadana ${ }^{6}$ subía y la vivienda escalaba rangos a partir de septiembre de 2000 (5,1\%) para no dejar de aumentar, realizando una carrera trepidante como tema o problema personal de los españoles, llegando a ocupar la tercera posición (17\%) en el mes de julio de 2003 y un segundo lugar (20,5\%) en febrero de 2004. La presencia de los problemas económicos en la agenda personal es notoria y figura con frecuencia entre los cuatro primeros rangos o posiciones.

Tal y como se observa en el Gráfico no 2 y el Gráfico no 3, se aprecia la evolución de los tres temas más relevantes en ambas agendas que mejor se posicionaron los meses más próximos a las elecciones de 2004. En la agenda personal se aprecia que el paro es el tema que no deja lugar a otros, de forma estable, seguido del terrorismo, aunque se contempla que la vivienda, a partir del abril del 2003, se posiciona en el tercer lugar, del que sólo desciende en septiembre del 2003 a un cuarto lugar o rango (13,3\%) para terminar de escalar a un segundo

\footnotetext{
${ }^{6}$ Para una mayor explicación de los movimientos del problema de la inseguridad ciudadana en ambas agendas se puede consultar el siguiente estudio monográfico: Rodríguez y Bouza (2007). La inseguridad ciudadana en las agendas pública y personal de los españoles (2000-2004) / Citizen Insecurity in the Public and Private Agenda of the Spanish Population (2000-2004). Política y Sociedad, 44(3), 183-196.
} 
lugar en febrero de 2004. Desde una perspectiva de agenda pública hay menos variaciones entre los tres temas, apreciándose la subida tímida de la vivienda y el descenso del terrorismo como preocupación pública a partir de julio de 2001.

\section{LAS AGENDAS PÚBLICA Y PERSONAL Y EL ÁREA DE IMPACTO DE LA COMUNICACIÓN POLÍTICA EN LAS ELECCIONES DEL 14-M. CONCLUSIONES PARA UN DEBATE.}

Las agendas pública y personal en el barómetro del CIS del mes de marzo ${ }^{7}$ de 2004, realizado del 16 al 21 de marzo, dos días después de las elecciones generales del 14 de marzo, muestran en la Tabla №1 la jerarquía en sus tres primeros temas.

TABLA nº1: Principales problemas en agenda pública y personal (marzo 2004)

\begin{tabular}{|c|c|c|c|}
\hline MARZO 2004 & PRIMER TEMA & SEGUNDO TEMA & TERCER TEMA \\
\hline AGENDA PÚBLICA & $\begin{array}{c}\text { Terrorismo, ETA } \\
(76,9 \%)\end{array}$ & $\begin{array}{c}\text { Paro } \\
(54,2 \%)\end{array}$ & $\begin{array}{c}\text { Vivienda } \\
(20,3 \%)\end{array}$ \\
\hline AGENDA PERSONAL & $\begin{array}{c}\text { Terrorismo, ETA } \\
(36,7 \%)\end{array}$ & $\begin{array}{c}\text { Paro } \\
(32,7 \%)\end{array}$ & $\begin{array}{c}\text { Vivienda } \\
(18,9 \%)\end{array}$ \\
\hline
\end{tabular}

Elaboración Propia. Fuente: CIS

Las diferencias en porcentajes tienen que ver con la diferente distribución de los temas en ambas agendas, pero no afectan a los rangos, que son coincidentes. Si asumimos la hipótesis del Área de Impacto $^{8}$ (a mayor coincidencia de tema y rango en ambas agendas, mayor incidencia o impacto de la comunicación política sobre esos temas: identificación plena de lo público y lo personal), podríamos decir que el Terrorismo asume una función central en la eficacia de la Comunicación Política, y que esa función central orienta el voto en ese sentido (político) que encuadra (framing) a los temas económicos (Paro, Vivienda), desplazándolos e interpretándolos desde la prioridad temática del terrorismo. Es decir: la acción terrorista del 11-M, moviendo la relevancia del terrorismo al primer rango de ambas agendas e incrementando sus porcentajes, tuvo una incidencia sustancial

\footnotetext{
7 Estudio № 2.558, marzo 2004, Centro de Investigaciones Sociológicas (CIS). http://www.cis.es/cis/export/sites/default/Archivos/Marginales/2540_2559/2558/ES2558.pdf (visita realizada el 18 de mayo de 2017).

8 Bouza, F. (2004). “The impact Area of Political Communication: Citizenship Faced with Public Discourse”, International Review of Sociology-Revue Internationale de Sociologie, Vol. 14, No. 2.
} 
en el encuadre (framing) del conjunto de la situación político-económica y desplazó al voto económico a favor del voto político.

El barómetro anterior al del mes de marzo (febrero del CIS ${ }^{9}$, realizado del 20 al 27 del mismo mes) muestra la siguiente distribución temática en la Tabla no2:

TABLA no 2. Principales problemas en agenda pública y personal

(febrero 2004)

\begin{tabular}{|c|c|c|c|}
\hline MARZO 2004 & PRIMER TEMA & SEGUNDO TEMA & TERCER TEMA \\
\hline AGENDA PÚBLICA & $\begin{array}{c}\text { Paro } \\
(65,6 \%)\end{array}$ & $\begin{array}{c}\text { Terrorismo, ETA } \\
(43,2 \%)\end{array}$ & $\begin{array}{c}\text { Inseguridad Ciu- } \\
\text { dadana }(22,9 \%)\end{array}$ \\
\hline AGENDA PERSONAL & $\begin{array}{c}\text { Paro } \\
(37 \%)\end{array}$ & Vivienda $(20,5 \%)$ & $\begin{array}{c}\text { Inseguridad } \\
\text { Ciudadana }(15,6 \\
\%)\end{array}$ \\
\hline
\end{tabular}

Elaboración Propia. Fuente: CIS

Las diferencias son obvias. La situación demoscópica, si tomamos la referencia más optimista hacia el Partido Popular en estimación de voto (Empresa TNS-Demoscopia, que trabajaba, entre otros, para la empresa del diario conservador $A B C$ ) mostraba una situación, en voto directo, que tuvo la evolución que muestra la Tabla no3 durante esos días ${ }^{10}$ :

\footnotetext{
${ }^{9}$ Estudio №2.556, febrero 2004, Centro de Investigaciones Sociológicas (CIS). http://www.cis.es/cis/export/sites/default/Archivos/Marginales/2540_2559/2558/ES2558.pdf (visita realizada el 18 de mayo de 2017).

${ }^{10}$ Informativos TELECINCO.com. Los datos del tracking estaban en la página web de Telecinco, para quien trabajó la empresa TNS-Demoscopia durante esos días electorales. Nuestras gestiones por hacernos con las matrices de esas encuestas no dieron resultado, pese al permiso de Telecinco.
} 
TABLA no ${ }^{3}$. Tracking voto directo PP/PSOE (8-13 marzo de 2004)

\begin{tabular}{|l|l|l|l|l|l|l|}
\hline $\begin{array}{c}\text { DÍAS/VOTO } \\
\text { DIRECTO } \\
\text { PP/PSOE }\end{array}$ & $8-\mathrm{M}$ & $9-\mathrm{M}$ & $10-\mathrm{M}$ & $11-\mathrm{M}$ & $12-\mathrm{M}$ & $13-\mathrm{M}$ \\
\hline PP & 21,1 & 26,8 & 23,9 & 24,1 & 23,6 & 20,7 \\
\hline PSOE & 20,6 & 19,1 & 24,9 & 15,1 & 18,9 & 19,7 \\
\hline
\end{tabular}

Elaboración propia. Fuente: Informativos Telecinco.com, TNS-Demoscopia.

El voto directo ha sido en los últimos años y elecciones, el mejor indicador de la tendencia. De alguna forma ha vuelto a serlo. Pero estos datos muestran también los efectos inmediatos del atentado: no tanto una subida del PP cuanto una bajada en voto directo del PSOE de 9,8 puntos de porcentaje. El terrorismo como tema interpretante de la situación (como marco o encuadre o encuadramiento framing-, pero también como tema inicial de toda interpretación inmediata priming-, con una relevancia muy grande) va a ir marcando la conducta de voto en dos sentidos: en primer lugar, y siguiendo la hipótesis clásica, a cualquier ataque contra el Estado se responde con un incremento de apoyo al Estado (representado en el Gobierno, aunque en este caso ese apoyo se hace "a contrario": negando votos al PSOE). Y, en segundo lugar, ante la gestión gubernamental del acto terrorista (probablemente, esta es la mejor forma de enunciar lo que realmente ocurrió esos días sin tener que recurrir al complejo y espinoso tema de la mentira, que fue la percepción popular mayoritaria ${ }^{11}$ ) se desplaza el apoyo al principal partido de la oposición. En todo caso, el encuadre (framing) del terrorismo debe ser central para interpretar la conducta electoral.

El Gráfico no 4 y el Gráfico no 5, sumados a la Figura A presentada al inicio de este artículo, expresan cuál fue la evolución de los principales problemas más importantes en la agenda pública y la personal de los meses enero, febrero, marzo y abril de 2004 y los nexos de conexión con el área de impacto de la comunicación política.

\footnotetext{
${ }^{11} \mathrm{Y}$ en los últimos días ¿cree usted que ha habido manipulación/ocultación de la información?:

SI: 64,7\%; NO: 24,7\%; NS: 10,5\%; NC: 0,1\%. Encuesta del "Pulsómetro", 22/3/2004. Cadena SER. http://cadenaser.com/ser/2004/03/22/espana/1079916611_850215.html (visita realizada el 18 de mayo de 2017).
} 


\section{Gráfico no 4}

Evolución Temas Importantes en Agenda Pública (rango)

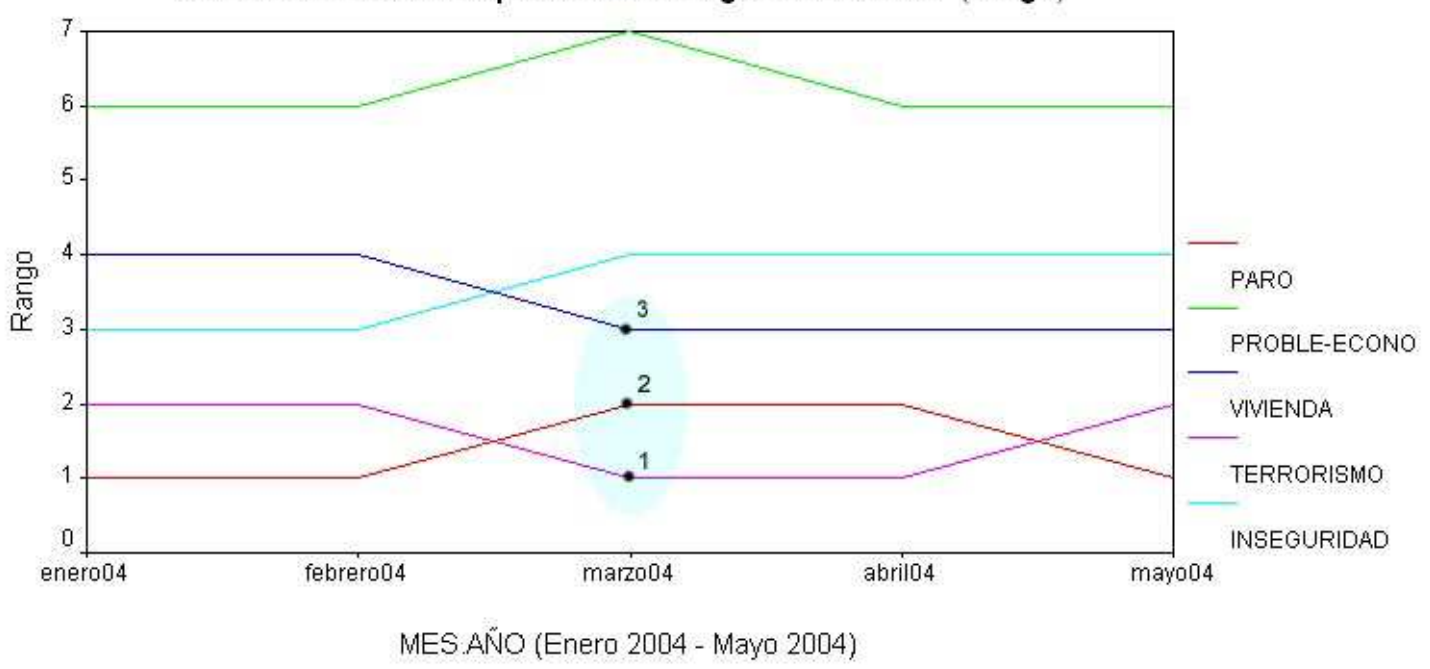

Elaboración Propia. Fuente: CIS

\section{Gráfico № 5}

Evolución temas principales en la Ag.Personal. (Rango)

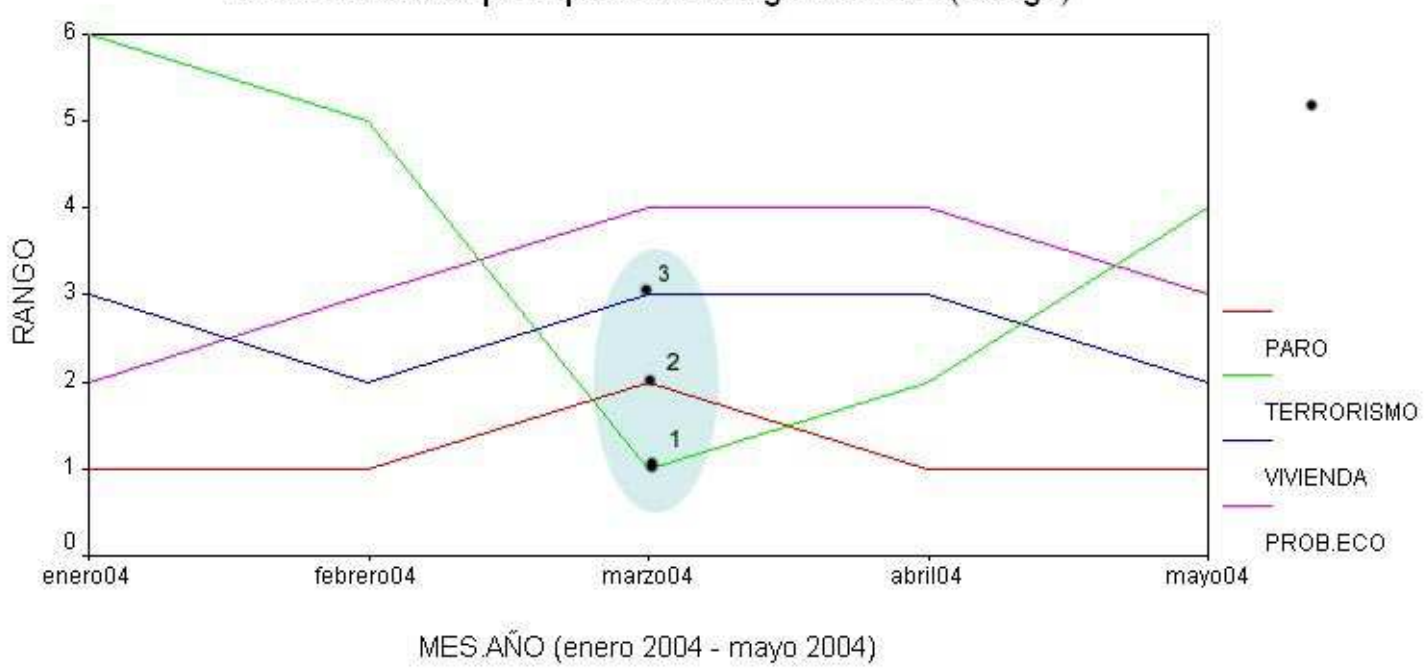

Elaboración Propia. Fuente: CIS

El terrorismo, el paro y la vivienda fueron los tres "temas estrella" de la comunicación política durante las elecciones de 2004, simbolizaron el encuentro de lo público y lo personal con la argumentación mixta de los individuos que actúan desde un área de impacto perfecta (coincidencia de temas y posición de los mismos en las dos agendas). Empleando otras palabras, podríamos decir que estos tres temas ocuparon el eje central de la comunicación política durante las elecciones españolas, ayudando a visibilizar en el debate público a aquellos partidos que mejor los posicionaran en su discurso. 


\section{REFERENCIAS.}

Álvarez De Toledo, C. (2004). Cuatro días de marzo: la verdad sobre el 11-M. Madrid: Planeta.

Johnson, A. J. (2002). Beliefs about arguing: A comparison of public issue and personal issue arguments, Communication Reports, pp. 99-111.

Joslyn, M.R. (1999). Perceiving public opinion of political figures: Examining the link between individual and collective opinion, International Journal of Public Opinion Research, fall 1999, pp. 213-232.

Blumler, J. G., \& McQuail, D. (1969). Television in politics: Its uses and influence. University of Chicago Press.

Bouza, F. (2004). The impact Area of Political Communication: Citizenship Faced with Public Discourse, International Review of Sociology - Revue Internationale de Sociologie, Vol. 14, nº 2, pp. 245- 259.

Bouza, F., González, J. J., Rodríguez, R., \& Castromil, A. (2008). “Voto racional y agenda mediática. Propuesta de seguimiento de la legislatura a través de grupos experimentales". En M. J. Canel y M. Gurrionero). Estudios de comunicación política. Libro del año 2008 (pp. 501-520). Madrid: Servicio de publicaciones de la Universidad Complutense.

Castromil, A., \& Rodríguez, R. (2011). Terrorismo con y sin tregua. Políticos, ciudadanos y medios de comunicación. Revista TELOS (87), pp. 46-56.

DeLuca, K., \& Peeples, J. (2002). From public sphere to public screen: Democracy, activism, and the" violence" of Seattle. Critical studies in media communication, 19(2), pp. 125-151.

Entman, R. M. (1993). Framing: Toward clarification of a fractured paradigm. Journal of communication, 43(4), pp. 51-58.

Entman, R. M. (2004). Projections of power: Framing news, public opinion, and US foreign policy. Chicago: University of Chicago Press.

González, J. J. (2002). Las elecciones generales del 2000. Voto ideológico / voto racional, Revista Internacional de Sociología, 32, pp. 7-33.

González, J. J., \& Bouza F. (2009). Las razones del voto en la España democrática, 1977-2008 (Vol. 296). Madrid: Los libros de la Catarata.

Lago, I, \& Montero, J. R. (2004). “Los mecanismos del cambio electoral. Del 11M al 14M", Claves de la razón práctica, 149, pp. 36-44.

Malalana, A. (2008). Aproximación historiográfica al 11-M (Vol. 4). Madrid: CEU

Edicio- 
nes.http://www.uspceu.com/instituto_democracia/pdf/publicaciones/doc umentos-trabajo/malalana.pdf (visita realizado el 5 de junio de 2017).

McCombs, M, \& Shaw, D. L. (1972). The Agenda-setting function of the mass media, Public Opinion Quarterly, n 36, pp. 176-187.

McCombs, M. (1999). Personal Involvement with issues on the public agenda, International Journal of Public Opinion Research, nº11, pp. 152 -168.

McCombs, M. (2004). Setting de Agenda. Cambridge, Polity Press.

Michavila, N. (2005). “Guerra, terrorismo y elecciones: incidencia electoral de los atentados islamistas de Madrid", Documento de trabajo no 13, 13 de marzo de 2005, Real Instituto Elcano, Madrid.

Olmeda, J. A. (2005). "Miedo o engaño: el encuadramiento de los atentados terroristas del 11-M en Madrid y la rendición de cuentas electoral", Real Instituto Elcano, 2005, no 70, pp.1-48.

Rodríguez. R. (2005). Miedo post 11-M y terrorismo en España. Recerca: revista de pensament $i$ anàlisi, (5), pp. 127-142.

Rodríguez, R. (2016). “Bases de datos para el análisis del contenido de la prensa: Atentado terrorista 2004 en Madrid" en M. Sánchez y C. Oliva, Formas de comunicación en el siglo XXI (pp. 209-227). Madrid: Dykinson.

Rodríguez, R., \& Bouza, F. (2007). La inseguridad ciudadana en las agendas pública y personal de los españoles (2000-2004) / Citizen Insecurity in the Public and Private Agenda of the Spanish Population (2000-2004). Politica y Sociedad, 44(3), pp. 183-196.

Rodríguez, R., \& Castromil, A. R. (2010). La circulación social de los encuadres periodísticos en tiempo de campaña electoral: Transmisión, influencia y atribución de responsabilidad. Zer-Revista de Estudios de Comunicación, 15 (29), pp. 193-212.

Sampedro Blanco, V. (2005). 13-M, Multitudes online. Madrid: Catarata.

Santamaría, J. (2004). “El azar y el contexto. Las elecciones generales de 2004”, Claves de la razón práctica, 146, octubre, pp. 28-43.

Swanson, D. L. (1995). El campo de la Comunicación Política. La democracia centrada en los Medios (pp. 3-24), en Muñoz-Alonso, A., \& Rospir, J. L. (eds), Comunicación Política. Madrid: Editorial Universitas.

Schulz, W. (1999). Television and declining political trust: How Germans react to changes in the media system. In International Communication Association Conference. San Francisco, CA.

Vara, A., Rodríguez, J., Giménez, E., \& Díaz, M., (Eds), (2006). Cobertura informativa del 11M. Pamplona: EUNSA. 


\section{WEBGRAFÍA}

Bouza, F. Página personal del Catedrático de Opinión Pública Fermín Bouza Álvarez, Departamento de Sociología VI, Facultad de Ciencias de la Información de la Universidad Complutense. http://pendientedemigracion.ucm.es/info/socvi/BOUZA/NUEVA1/Textos /impactesp.pdf (visita realizada el 7 de julio de 20017).

Centro de Investigaciones Sociológicas (CIS). Estudio №2.556, febrero 2004, Centro de Investigaciones Sociológicas (CIS). http://www.cis.es/cis/export/sites/default/archivos/Marginales/2540_2559 /2558/ES2558.pdf (visita realizada el 18 de mayo de 2017).

Centro de Investigaciones Sociológicas (CIS). Estudio № 2.558, marzo 2004, Centro de Investigaciones Sociológicas (CIS). http://www.cis.es/cis/export/sites/default/Archivos/Marginales/2540_255 9/2558/ES2558.pdf (visita realizada el 18 de mayo de 2017).

La Vanguardia, 7 de marzo de 2004. http://hemeroteca.lavanguardia.com/preview/2004/03/07/pagina1/33651159/pdf.html (visita realizada el 7 de mayo de 20017).

Pulsómetro, encuesta, 22/3/2004. Cadena SER. http://cadenaser.com/ser/2004/03/22/espana/1079916611_850215.html (visita realizada el 18 de mayo de 2017).

FERMín BOUZA ÁLVAREZ. Catedrático de Opinión Pública en la Universidad Complutense de Madrid. Investigador y escritor, especializado en el estudio de los medios de comunicación, la realidad política y el comportamiento electoral.

RAQUEL RODRÍGUEZ DÍAZ. Profesora e investigadora en la Universidad Rey Juan Carlos, con una extensa experiencia en investigación de Agenda Setting y su aplicación empírica en diferentes ámbitos.

Recibido: 10/04/2017

Aceptado: 01/11/2017 